\section{O4E.3 UTILITY OF ROUTINELY RECORDED DATA ON WORKING HOURS FOR AN EPIDEMIOLOGICAL COHORT STUDY OF 60,000 SWEDISH HEALTH CARE EMPLOYEES}

\footnotetext{
1,2Per Gustavsson* ${ }^{1,2}$ Carolina Bigert, ${ }^{1,2}$ Theo Bodin, 'Jenny Selander, ${ }^{3}$ Mikko Härmä, ${ }^{1,2}$ Annika Lindahl-Norberg, ${ }^{1,2}$ Tomas Andersson, ${ }^{1,2}$ Annika Gustavsson, ${ }^{1,2}$ Maria Albin. ${ }^{1}$ Institute of Environmental Medicine, Karolinska Institutet, Stockholm, Sweden; ${ }^{2}$ Centre for Occupational and Environmental Medicine, Stockholm County Council, Stockholm, Sweden; ${ }^{3}$ Finnish Institute of Occupational Health, Helsinki, Finland
}

\subsection{6/OEM-2019-EPI.108}

Night and shift work is common in industry and service that must be functional 24 hour. Night work disrupts the circadian rhythm, and is potentially linked to an excess of cancer (especially breast cancer), cardiovascular disease, pregnancy complications, as well as to psychical effects. Large studies with detailed and objectively collected data on working hours are needed to confirm causal associations and develop practical advice on how shift schedules should be planned in order to minimize negative health effects.

Stockholm County Council employs a high number of health care workers of which many are working in shifts. We have formed a cohort of those employed from 2008 to 2016, 45000 women and 15000 men. The computerized employment register holds individual records on working hours day by day in retrospect since 2008, and information on night work since year 2000. Information on cancer incidence, hospital discharge diagnoses of cardiovascular disease, pregnancy outcome, and causes of sick-leave have been obtained from national central registers.

There were typically three work shifts: morning shifts (0715), afternoon shifts (14-21), and night shifts (21-07). Non shift-workers typically worked (08-17). So far, we have investigated work shift patterns for those with a high percentage of night work: nurses, assistant nurses and midwives. Among these, 22\% worked at least one night per month, and $15 \%$ worked at least 5 nights per month in 2009, which increased slightly to $23 \%$ and $16 \%$ respectively in 2016 . It was common to work several nights in a row: in 2009 13.8\% worked at least 3 consecutive nights or more per month, which increased to $15.5 \%$ in 2016 . Only $0.6 \%$ worked 5 consecutive nights or more per month.

Analyses of cancer, pregnancy outcome, cardiovascular disease and psychical outcomes are planned for 2019.

\section{E.4 APPLICATION OF PROBABILISTIC BIAS ANALYSIS TO ADJUST FOR EXPOSURE MISCLASSIFICATION IN A COHORT OF TRICHLOROPHENOL WORKERS}

Laura Scott*, George Maldonado. University of Minnesota School of Public Health, Minneapolis, USA

\subsection{6/OEM-2019-EPI.109}

This method was developed to demonstrate the application of probabilistic bias analysis to quantify and adjust for exposure misclassification in a historical cohort mortality study of New Zealand trichlorophenol workers where exposure to 2,3,7,8-tetrachlorodibenzo-p-dioxin (TCDD) was measured as a multi-level variable. Published exposure information available for this cohort of workers was used to specify the initial bias parameter distributions, which were then varied under 18 different scenarios to assess the potential impact of differing amounts of misclassification as well as both non-differential and differential exposure misclassification. For each scenario, each bias parameter distribution was sampled 50000 times using Monte Carlo simulation techniques to generate adjusted counts of cases and non-cases of ischemic heart disease (IHD) by exposure group. These counts were then used to calculate odds ratios adjusted for exposure misclassification and the associated exposure misclassification error terms. Given the specified assumptions, the geometric mean (GM) adjusted odds ratio had a range of 2.89 to 5.05, and the GM error term ranged from 0.60 to 1.05 . In all non-differential scenarios and scenarios in which non-cases had greater proportions of misclassification, the observed odds ratio of 3.05 was closer to the null (i.e., 1) than the GM adjusted odds ratio. For the differential simulations where cases had higher proportions of misclassification, the direction of the error was dependent on the level of misclassification error, with smaller proportions of misclassification resulting in the observed odds ratio being farther away from the null than the GM adjusted odds ratio. These findings demonstrate that probabilistic bias analysis of historical cohort mortality studies can be an effective tool for understanding trends in study error stemming from exposure misclassification and confirm the importance of quantifying potential sources of systematic error.

\section{O4E.5 ABSTRACT WITHDRAWN}

\section{Musculoskeletal Disorders-2}

\section{A.1 DEFINITION OF CASE MANAGEMENT FOR RETURN-TO- WORK IN THE APPROACH OF WORKERS WITH MUSCULOSKELETAL DISORDERS: AN UPDATE USING A SCOPING REVIEW}

\begin{abstract}
1,2,3 Mercè Soler Font*, ${ }^{*}, 2,3$ José Maria Ramada Rodilla, ${ }^{4,5,6}$ José Maria Gonzalez, ${ }^{7,8}$ Francisco Palencia Sanchez, ${ }^{1,2,3}$ Consol Serra Pujadas. 'Center for Research in Occupational Health, Department of Experimental and Health Sciences University Pompeu Fabra, Barcelona, Spain; ${ }^{2}$ IMIM (Hospital del Mar Medical Research Institute), Barcelona, Spain; ${ }^{3}$ CIBER of Epidemiology and Public Health, Barcelona, Spain; ${ }^{4}$ Public Health and Preventive Medicine Training Unit, Parc de Salut Mar, Barcelona, Spain; ${ }^{5}$ Public Health Agency of Barcelona, Barcelona, Spain; ' University Pompeu Fabra, Barcelona, Spain; 7Universidad Nacional de Colombia, Doctorado Interfacultades en Salud Pública, Bogotá, Colombia; ${ }^{8}$ Pontificia Universidad Javeriana, Facultad de Medicina, Departamento de Medicina Preventiva y Social, Bogotá, Colombia
\end{abstract}

\subsection{6/OEM-2019-EPI.110}

Purpose We aim to identify the elements and functions that define the case management in the individual approach of workers with musculoskeletal disorders for return to work and to propose an updated definition.

Methods Relevant articles were identified through a computerized search up to 1 December 2017 in the bibliographic databases PubMed, Web of Science, Scopus, Cochrane Library, IBECS, EMBASE, and LILACS. Language filter (English and Spanish) was included in the search. The inclusion criteria were working age population with musculoskeletal disorders, studies that described or evaluated case management interventions, and return to work interventions. Military personnel, and rheumatic and surgical pathologies were excluded. 
Independent pairs reviewed all cites and articles and extracted data, and narrative synthesis was carried out.

Results Our searches identified 1950 possible relevant articles, of which 27 were included for data extraction. Of these, 15 papers included definitions of case management, and 14 common elements were identified. Twenty two explained the role of case manager and in 18 a description of tasks was found. In 25 articles referral services were mentioned. The most common defining elements were 'return to work intervention', 'multidisciplinary assessment' and 'interdisciplinary intervention' $(53.8 \%)$. The $40 \%$ of the articles emphasized the 'coordination', 'to influence multiple factors' (33.3\%) and 'individual approach' (26.7\%). The tasks included 'to establish goals and plan the rehabilitation for return to work' $(50 \%)$, and 'supervise or coordinate return to work process and to offer/refer the employee to services/adaptations or therapeutic workplaces' (36.4\%) The most common offered services were mental health $(64.0 \%)$, and rehabilitation (48.0\%).

Conclusions Despite the increase in the number of published articles dealing with case management, usually it is not clearly defined. This scoping review emphasizes the need to define case management and its organizational characteristics, and proposes an extended and updated definition.

\section{A.2 MUSCULOSKELETAL SYMPTOMS AND WORK-RELATED ACCIDENTS: A HOSPITAL-BASED CASE-CONTROL STUDY}

${ }^{1}$ Adriano Dias*, ${ }^{1}$ João Marcos Bernardes, ${ }^{2,3}$ Juan Gomez-Salgado, ${ }^{2,3}$ Carlos Ruiz-Frutos. ${ }^{1}$ Unesp - São Paulo State University, Botucatu Medical School, Botucatu, Brazil; ${ }^{2}$ Universidad de Huelva, Huelva, Spain; ${ }^{3}$ Universidad Espíritu Santo, Guayaquil, Ecuador

\subsection{6/OEM-2019-EPI.111}

This hospital-based case-control study aimed to determine whether self-reports of musculoskeletal symptoms (MSS) were associated with the occurrence of work-related accidents. Study participants were recruited from the emergency department at Botucatu Medical School University Hospital. Cases were workers who suffered work-related accidents that required hospitalization, while controls were selected patients who suffered a non-work-related accident. Participants were interviewed using a standardized structured questionnaire with close-ended questions and a modified version of the Brazilian Portuguese Nordic Musculoskeletal Questionnaire. Associations between self-reports of MSS and work-related accidents were analyzed with two logistic regression models (one for symptoms that occurred in the 12 months period and the other for those that occurred in the previous 7 days). These analyses were performed in two steps: univariate and multiple model. Variables with a Pvalue $\leq 0.25$ in the univariate analysis were included in the multiple models, using the forward stepwise selection procedure. In the multiple models two-sided P-values less than 0.05 were considered statistically significant. Altogether, 80 cases and 125 controls were included. The participants had a mean age of 36.9 years $(\mathrm{SD}=11.4)$ and $72.2 \%$ were men. In the 12 months multiple logistic regression model, self-report of MSS in the upper limbs (OR 2.689 95\% CI 1.357-5.326) was associated with increased odds of workrelated accidents occurrence, while in the 7 days multiple logistic regression model, self-report of MSS in the upper limbs (OR 2.374 95\% CI 1.083-5.201) and in the vertebral column (OR 2.154 95\% CI 1.017-4.561) were associated. Thus, this case-control study suggests that MSS in the upper limbs and in the vertebral column are associated with increased odds of work-related accidents; and that the Nordic Musculoskeletal Questionnaire may be used as a complementary screening tool for identifying workers at risk for work-related accidents.

\section{A.3 INJURED AT WORK: FACTORS PREDICTIVE OF FURTHER WORK-RELATED INJURIES - A PROSPECTIVE STUDY}

${ }^{1}$ Helen Harcombe*, ${ }^{2}$ Ari Samaranayaka, ${ }^{3}$ Emma H Wyeth, ${ }^{1}$ Gabrielle Davie, ${ }^{4}$ lan D Cameron, ${ }^{1}$ Sarah Derrett. ${ }^{1}$ Injury Prevention Research Unit, Department of Preventive and Social Medicine, University Of Otago, New Zealand; ${ }^{2}$ Ng̈̈ Tahu Mæri Health Research Unit, Department of Preventive and Social Medicine, University of Otago, New Zealand; ${ }^{3}$ John Walsh Centre for Rehabilitation Research, Kolling Institute, University of Sydney, Australia; ${ }^{4}$ Biostatistics Unit, Dean's Department, University of Otago, New Zealand

\subsection{6/OEM-2019-EPI.112}

Background Work-related injuries can have a large impact on employees, employers and wider society. Preventing subsequent work-related injuries from occurring among those who have already had a work-related injury has the potential to reduce the burden of work-related injuries. However, predictors of subsequent work injuries must first be understood.

This study investigates New Zealand participants from the Prospective Outcomes of Injury Study (POIS) with a substantial work-related injury (the 'sentinel' injury) and examines subsequent work-related injury events occurring during the following 24 months. Objectives are to: 1) describe the nature of sentinel and subsequent work-related injuries, and 2) examine work-related predictors of subsequent workrelated injuries.

Methods Of the 2856 POIS participants, 754 were recruited following a substantial work-related injury. Data were combined from: 1) participant interviews approximately 3 months following their sentinel injury, 2) administrative claims data from New Zealand's universal no-fault injury insurer (the Accident Compensation Corporation), and 3) hospital discharge data to 24 months. Predictors of subsequent work-related injuries were examined using multivariable analyses.

Results Work-related sentinel injury events most commonly involved spine dislocations/sprains/strains (25\%) with 'lifting/ loading/pulling' the most common work task involved. One third $(34 \% ; n=258)$ went on to have at least one subsequent work-related injury in the following 24 months. Of those whose sentinel work-related injury was related to 'lifting/loading/pulling', $19 \%$ had at least one subsequent work-related injury event also related to this type of task. Predictors examined included pre-sentinel injury job strain, social support from colleagues and supervisors, physical work tasks, job security and job satisfaction.

Conclusion A substantial proportion of participants with a work-related sentinel injury had further work-related injuries in the following 24 months. The identification of factors that predict those at risk of subsequent work-related injuries could provide a useful focus for those involved in the rehabilitation of people with work-related injuries. 